\title{
U.S. Cohort Differences in Body Composition Outcomes of a 6-Month Pedometer-Based Physical Activity Intervention: The ASUKI Step Study
}

\author{
Jenelle R. Walker ${ }^{1, *}$; Ali Soroush ${ }^{2}$; Barbara E. Ainsworth ${ }^{3}$; Michael Belyea ${ }^{1}$; Pamela D. Swan \\ ${ }^{3}$; Agneta Yngve ${ }^{4}$ \\ ${ }^{1}$ College of Nursing and Health Innovation, Arizona State University, Phoenix, AZ, USA \\ ${ }_{2}^{2}$ Department of Sports Medicine and Rehabilitation, Imam Reza Hospital, Kermanshah University of Medical Sciences (KUMS), Kermanshah, IR Iran \\ ${ }^{3}$ School of Nutrition and Health Promotion, Arizona State University, Phoenix, AZ USA \\ ${ }^{4}$ Department of Nursing, Sophiahemmet University, Stockholm, Sweden \\ ${ }^{*}$ Corresponding author: Jenelle R. Walker, College of Nursing and Health Innovation, Arizona State University, 500 N. Third Street, Phoenix, AZ, USA. Tel: 602-4962644, Fax: 602- \\ 4960886, E-mail: jenelle.walker@asu.edu
}

Received: July 12, 2013; Accepted: February 25, 2014

Background: Regular physical activity(PA) enhances health and is an important factor in disease prevention and longevity. The 2008 U.S. Physical Activity Guidelines recommends that all healthy adults attain at least 150 minutes per week of moderate intensity aerobic PA(e.g., brisk walking) to maintain and promote PA.

Objectives:This study determined the effects of a 6-month pedometer-based worksite walking intervention with participants focusing on a goal of achieving 10,000 steps per day, on body composition in adults with a wide range of body mass index (BMI) values and compares the changes with outcomes of similar studies.

Materials and Methods: The design was a single group, quasi-experimental study. All participants received a pedometer and were asked to register the daily number of steps. Men and women $\left(\mathrm{n}=142\right.$; age $=41 \pm 11.5$ years; $\left.\mathrm{BMI}=27.2 \pm 7.25 \mathrm{~kg} \cdot \mathrm{m}^{-2}\right)$ received body composition measures at 1,3, and 6 months. A multilevel growth modeling approach was used to explore change over time and to predict change by steps, age, gender, and fat category categorized as normal and overweight/obese.

Results: Significant individual differences in linear slopes and change over time were observed for waist circumference $(\mathrm{WC})(-3.0 \mathrm{~cm})$ only in unconditional model $(\mathrm{t}=-0.67, \mathrm{P}=0.02)$.

Conclusions: A $3.0 \mathrm{~cm}$ loss in WC shows that a 10,000 step per day walking program has the potential to influence changes in body composition measures that are correlated with adverse health outcomes. While significant changes did occur there are some limitations. The analysis did not consider the data regarding completing of 10,000 steps per day and other potential factors that could influence the results. Compliance to the walking dose and initial physical activity and body composition levels are important to consider when studying body composition changes in such programs.

Keywords:Work site, Intervention; Walking; Waist Circumference; Body Fat; Physical Activity

\section{Background}

Regular physical activity (PA) enhances health and is an important factor in disease prevention and longevity. The 2008 U.S. Physical Activity Guidelines recommends that all healthy adults attain at least 150 minutes per week of moderate intensity aerobic PA (e.g., brisk walking) to maintain and promote PA (1). The guidelines further recommend that PA be doubled for added health benefits such as prevention of weight gain and weight maintenance. However, most Americans do not reach the recommended levels of PA (2) and, the fact that $35.7 \%$ of Americans have a body mass index (BMI) in the obese category increases the salience of regular PA as a health enhancing behavior $(3,4)$. Worksite nutrition and PA programs for obesity prevention and control have been identified by the Guide to Community Preventive Services (5) as an effective way to improve health-related be- haviors and health outcomes. Pedometer-based 10,000 steps per day walking programs have been used globally to promote increased walking for health, including modifying body composition, improving cardiorespiratory fitness, and reducing risk factors related to metabolic syndrome (6-8).

While such programs have a standard goal to walk 10,000 steps, which at a rate of 100 steps per minute equal to about 100 minutes of brisk walking per day, changes in body weight, fat percent fat, WC and other body composition measures are mixed (6-9). Some programs show no changes in body composition values (6, 7) while others show only modest losses in body weight and WC (8) with the lengths of the programs ranging from 8 to 24 weeks. In a meta-analysis of pedometer intervention programs, Bravata et al. (9) randomized con- 
trolled trials and observational studies ranged from 3 to 104 weeks with an estimated the average weight loss for the 10,000 steps/day programs of approximately $0.38 \mathrm{~kg}$ in body weight. In a 4-month pedometer-based worksite health program male and female participants had a $1.6 \pm 5.9 \mathrm{~cm}$ average reduction in WC (6). Interestingly, in Swedish women enrolled in the 6-month ASUKI Step pedometer-based worksite walking program, Soroush et al. (10) showed small, but statistically significant reductions in waist circumference in (-0.84 cm every 3 months) and BMI (-0.24 units every three months). This finding resulted in spite of the fact that $63.5 \%$ of the sample had a $\mathrm{BMI}$ in the normal category. A reduction in waist circumference is interesting among normal weight individuals due to its singularly close relationship to metabolic diseases.

Encouraging overweight/obese adults to engage in walking programs with a dose of activity that meets the US Physical Activity Guidelines has important personal and public health consequences at a cadence of 100 steps per minute (11). Intentionally walking for 30-60 minutes per day requires approximately 3,000-6,000 steps. Adding this volume of walking to one's daily routine, which accounts for approximately 4000-5000 steps per day, can provide modest weight and body composition changes associated chronic disease reduction (12-15).

There are few comparative studies using the 10,000 steps/day walking interventions with the same study design and study procedures. The ASUKI Step study was a 6-month, pedometer-based 10,000 steps/day walking intervention with employees from Arizona State University (US cohort) in the greater Phoenix area, Arizona, USA, and the Karolinska Institutet (Swedish cohort) in Stockholm, Sweden. The study used social support theory to guide teams of 3 or 4 persons to encourage each other to walk 10,000 steps per day. Health outcome data for a sub-sample of study participants were collected to assess changes in pedometer and accelerometer-based physical activity, cardiovascular fitness, and body composition. The unique aspect of the ASUKI Step sub-sample were the differences in initial body composition values between the US (BMI $\left.=27.2 \pm 7.2 \mathrm{~kg} . \mathrm{m}^{-2}\right)$ and Swedish $(\mathrm{BMI}=24.1 \pm$ $\left.3.6 \mathrm{~kg} \cdot \mathrm{m}^{-2}\right)$ cohorts.

\section{Objectives}

By analyzing changes in body composition measures separately in each cohort, who both who completed the same study protocol, we can compare the impact of different initial body composition on changes over time.

\section{Materials and Methods}

To examine changes in pedometer steps taken each month and assess the relationship between average number of steps per day and body composition outcomes in the US cohort, we used the same analytic procedures described by Soroush et al. (10) for the Swedish cohort. The primary hypothesis was that the US cohort would show decreases in body mass index (BMI), percent body fat (BF), waist circumference (WC), and sagittal abdominal diameter (SAD). The secondary hypothesis was that specific predictor variables [age, gender, steps taken, fat category (normal and overweight/obese)] would predict the changes in the body composition outcomes.

\subsection{Overview of the Study}

The ASUKI Step study was a collaborative, six-month pedometer-based workplace intervention project held on the campuses of Arizona State University (ASU) and the Karolinska Institutet (KI) with an overall goal to increase physical activity by walking 10,000 steps per day. Participants were organized in teams of three to four persons to promote social support for walking and instructed to register the daily number of steps and/or step equivalents (half an hour of bicycling, aerobics, etc. rendered 2,700 steps) on a website at the end of each day. The complete ASUKI Step methods and procedures have been described in more detail elsewhere (16).

\subsection{Setting and Sample}

This study was a six month quasi-experimental design with measures taken at three intervals (months 1 , 3 , and 6; no control group). A total of 712 university faculty, staff, and graduate students from ASU, aged 20 to 70 years, participated in the study. A sub-sample of 142 randomly selected participants (32 men and 110 women and, referred to as the US cohort), completed a set of laboratory tests to determine physiological and anthropometric changes during the study. All participants gave written informed consent before inclusion in the study. The study was approved by the Arizona State University Institutional Review Board.

\subsection{Sources of Data}

Instruments used to obtain study data are described in detail by Ainsworth et al. (16) and Soroush et al. (10). Demographic characteristics were obtained from a webbased questionnaire developed for the ASUKI Step study. Daily steps were recorded by a Yamax pedometer SW 200 (Yamax Corporation, Tokyo, Japan) and were recorded on the study website. Body weight (kg) and BF were determined by bioelectrical impedance analysis with a Tanita TBF-300A scale (TANITA Corporation, Arlington Heights, IL) (17). All values were measured in duplicate with deviations greater than $0.1 \mathrm{~cm}$ for SAD and WC and greater than $0.2 \mathrm{~cm}$ for height were being repeated a third time.

\subsection{Data Analysis}

Data were analyzed using descriptive statistics, including frequency, means, and standard deviations (SD), to describe participant characteristics. All variables were checked for normality. A multilevel growth modeling 
WalkerJR et al.

approach was used to explore change over time and predict change by steps, age, gender, and fat categories [collapsed to two categories (normal and overweight/ obese)] for body composition measures. To categorize the predictor variable, fat category, the age-adjusted body fat percentage index for men and women was used, according to the cut-offs recommended by Gallagher et al. (18).

The multilevel approach answered three questions: (a) what is the change over time trajectory for the entire group (answered by the fixed effects of the unconditional model)?; (b) whether a distinct trajectory for each individual in the entire group is warranted (answered by the random effects in the model which is the slope and covariance between them)?; and (c) are there variables that predict these trajectories for the entire group? This is answered by the unconditional model by first establishing the functional form of change over time (linear or quadratic) and whether individual trajectories are needed and second by the final conditional mixed model to look at the predictors of the trajectories.

Each body composition measure was analyzed using four models: unconditional model (1), in conditional models [age (2), gender (3)] and in a final conditional mixed model (4) (steps taken, age groups, gender, and body fat percentage category (normal and overweight/ obese) as predictors of body composition changes). Statistical intent-to-treat analyses using all available data were performed using SAS software version 9.2. The level of significance was set at $\mathrm{p}<0.05$ for all tests. Following recommendations of Cohen and Cohen (1983), to further explore significant predictors estimates where calculated 1 SD above and 1 SD below (19).

\section{Results}

Descriptive characteristics of the US cohort are shown in Table 1 . The retention rate for completing body composition measures was $68.3 \%$. The results from the multilevel models are described below.

The results from the multilevel growth modeling analyses are described separately; with the unconditional analyses output presented in Table 2 and the final conditional analyses mixed model output presented in Table 3.

Table 1. Combined Body Composition Measures and Steps from Months 0-3 ${ }^{a}$

\begin{tabular}{|c|c|c|c|}
\hline Parameter & Month 0 & Month 3 & Month 6 \\
\hline \multicolumn{4}{|l|}{ Gender, No. } \\
\hline Female & 110 & 99 & 77 \\
\hline Male & 32 & 28 & 20 \\
\hline Total & 142 & 127 & 97 \\
\hline \multicolumn{4}{|c|}{ Body Mass Index, kg.m² } \\
\hline & $26.4 \pm 6.4$ & $26.8 \pm 7.9$ & $25.1 \pm 5.0$ \\
\hline & $29.9 \pm 9.2$ & $29.4 \pm 9.7$ & $29.6 \pm 10.8$ \\
\hline & $27.2 \pm 7.2$ & $27.4 \pm 8.3$ & $26.0 \pm 6.8$ \\
\hline \multicolumn{4}{|c|}{ Percent Body Fat } \\
\hline & $33 \pm 8.37$ & $32.4 \pm 7.9$ & $31.7 \pm 7.5$ \\
\hline & $26.4 \pm 12.7$ & $25.7 \pm 13.1$ & $24.8 \pm 13.0$ \\
\hline & $31.5 \pm 9.9$ & $30.9 \pm 9.7$ & $30.3 \pm 9.3$ \\
\hline \multicolumn{4}{|c|}{ Waist Circumference, cm } \\
\hline & $87.5 \pm 15.4$ & $85.9 \pm 14.9$ & $83.9 \pm 12.2$ \\
\hline & $100.2 \pm 25.1$ & $99.1 \pm 21.9$ & $100.7 \pm 25.8$ \\
\hline & $90.2 \pm 18.7$ & $88.5 \pm 17.4$ & $87.2 \pm 17.2$ \\
\hline \multicolumn{4}{|c|}{ Sagittal Abdominal Diameter, cm } \\
\hline & $19.5 \pm 3.9$ & $19.6 \pm 3.9$ & $18.8 \pm 3.4$ \\
\hline & $22.3 \pm 5.3$ & $22.6 \pm 5.4$ & $22.3 \pm 6.8$ \\
\hline & $20.1 \pm 4.4$ & $20.1 \pm 4.4$ & $19.5 \pm 4.5$ \\
\hline \multicolumn{4}{|l|}{ Steps, day } \\
\hline & $10,513.9 \pm 3,258.5$ & $8,484.5 \pm 4,831.1$ & $6,191.8 \pm 5,420.0$ \\
\hline & $11,461.8 \pm 3,665.0$ & $9,039.6 \pm 5,453.8$ & $6,857.1 \pm 6,012.6$ \\
\hline & $10,714.23,357.4$ & $8,601.8 \pm 4954.1$ & $6,332 \pm 5535.0$ \\
\hline
\end{tabular}

\footnotetext{
a data are presented as Mean \pm SD.
} 
WalkerJR et al.

\begin{tabular}{|c|c|c|c|c|c|}
\hline Parameter & Intercept & Time & Variable Intercept & Variable Slope & Covariance Intercept and Slope \\
\hline \multicolumn{6}{|l|}{ Body Mass Index } \\
\hline Coefficient & 27.2 & -0.02 & 46.09 & 0.00 & 6.90 \\
\hline Standard Error & 0.62 & 0.19 & 6.77 & 0 & 3.19 \\
\hline Pvalue & $0.0001^{\mathrm{a}}$ & 0.9 & $0.0001^{\mathrm{a}}$ & 0 & 0.03 \\
\hline \multicolumn{6}{|l|}{ Percent Body Fat } \\
\hline Coefficient & 31.5 & -0.24 & 95.59 & 0.69 & -3.29 \\
\hline Standard Error & 0.83 & 0.12 & 11.66 & 0.35 & 1.34 \\
\hline Pvalue & $0.0001^{\mathrm{a}}$ & 0.06 & $0.0001^{\mathrm{a}}$ & 0.03 & 0.04 \\
\hline \multicolumn{6}{|c|}{ Waist Circumference } \\
\hline Coefficient & 90.2 & -0.67 & 330.83 & 1.52 & -15.18 \\
\hline Standard Error & 1.56 & 0.28 & 40.88 & 1.48 & 5.61 \\
\hline Pvalue & $0.0001^{\mathrm{a}}$ & 0.02 & 0.0001 & 0.15 & 0.007 \\
\hline \multicolumn{6}{|c|}{ Sagittal Abdominal Diameter } \\
\hline Coefficient & 20.08 & -0.26 & 18.18 & 0.12 & 0.61 \\
\hline Standard Error & 0.37 & 0.08 & 2.29 & 0.13 & 0.39 \\
\hline Pvalue & $0.0001^{\mathrm{a}}$ & 1 & $0.0001^{\mathrm{a}}$ & 0.2 & 0.1 \\
\hline
\end{tabular}

a Statistical intent-to-treat analyses using all available data were performed using SAS software version 9.2. The level of significance was set at $\mathrm{P}<0.05$ for all tests. Time variable is for changes from T1 (beginning of study) to T3 (end of study).

\begin{tabular}{|c|c|c|c|c|c|c|c|c|c|c|c|c|}
\hline \multirow[b]{2}{*}{ Effect } & \multicolumn{3}{|c|}{ Body Mass Index } & \multicolumn{3}{|c|}{ Waist Circumference } & \multicolumn{3}{|c|}{ Percent Body Fat } & \multicolumn{3}{|c|}{$\begin{array}{c}\text { Sagittal Abdominal } \\
\text { Diameter }\end{array}$} \\
\hline & Coefficient & SE & P. value & Coefficient & SE & P. value & Coefficient & SE & P. value & Coefficient & SE & P. value \\
\hline Intercept & 32.1 & 1.45 & 0.0001 & 102.8 & 3.13 & 0.0001 & 29.9 & 1.43 & $0.0001^{\mathrm{a}}$ & 23.9 & 0.75 & $0.0001^{\mathrm{a}}$ \\
\hline Time & -0.31 & 0.66 & 0.6 & 0.48 & 1.04 & 0.6 & -0.78 & 0.45 & 0.09 & -0.23 & 0.28 & 0.4 \\
\hline Steps & 0 & 0 & 0.4 & 0 & 0.0001 & 0.6 & 0 & 0 & 0.1 & 0 & 0 & 0.1 \\
\hline Cage & 0.03 & 0.04 & 0.45 & 0.18 & 0.11 & 0.1 & 0.11 & 0.05 & $0.04^{\mathrm{a}}$ & 0.05 & 0.03 & 0.09 \\
\hline Gender & -1.77 & 1.28 & 0.2 & -7.67 & 3.12 & $0.02^{\mathrm{a}}$ & 9.27 & 1.47 & $0.0001^{\mathrm{a}}$ & -1.72 & 0.72 & $0.02^{\mathrm{a}}$ \\
\hline FatCat & -7.53 & 1.12 & 0.0001 & -20.23 & 2.72 & $0.0001^{\mathrm{a}}$ & -12.9 & 1.3 & $0.0001^{\mathrm{a}}$ & -4.9 & 0.63 & $0.0001^{\mathrm{a}}$ \\
\hline Time $^{\mathrm{a}}$ steps & 0 & 0 & 0.4 & 0 & 0 & 0.1 & 0 & 0 & 0.9 & 0 & 0 & 0.7 \\
\hline Time ${ }^{a}$ Cage & -0.01 & 0.02 & 0.7 & -0.03 & 0.03 & 0.3 & -0.002 & 0.01 & 0.9 & -0.01 & 0.01 & 0.5 \\
\hline Time $^{\mathbf{a}}$ Gender & -0.18 & 0.49 & 0.7 & -0.63 & 0.7 & 0.4 & 0.2 & 0.31 & 0.4 & 0.04 & 0.2 & 0.8 \\
\hline Time $^{\mathbf{a}_{\text {FatCat }}}$ & -0.07 & 0.43 & 0.9 & 1.2 & 0.61 & 0.05 & 0.47 & 0.27 & 0.09 & 0.17 & 0.18 & 0.3 \\
\hline
\end{tabular}

\footnotetext{
a Cage: Centered Age; FatCat: Normal vs. Overweight/obese; SE: Standard Error. Statistical intent to treat analyses using all available data was performed using SAS software version 9.2. The level of significance was set at $\mathrm{P}<0.05$ for all tests. Time variable is for changes from $\mathrm{T} 1$ (beginning of study) to T3 (end of study).
}

\subsection{Body Mass Index (BMI)}

There was no overall significant change in BMI following the intervention though at baseline, BMI was $27.2 \pm$ $7.2 \mathrm{~kg} \cdot \mathrm{m}^{-2}$ and $26.0 \pm 6.8 \mathrm{~kg} \cdot \mathrm{m}^{-2}$ at month 6 . Older participants (conditional model 2) had higher initial BMI values, where as age increased, the initial BMI value increased. There was no significant change in BMI over time for by age. There were significant gender differences (conditional model 3) in the starting BMI. Females on average were $3.57 \mathrm{~kg} . \mathrm{m}^{-2}$ smaller than males. In the final conditional mixed model (4) that included gender, age, steps, and percent fat category, the gender and age differences were no longer significant. However, those who had normal fat percent fat (BF) had initial BMI's that were $-7.53 \mathrm{~kg} \cdot \mathrm{m}^{-2}$ smaller than overweight/obese participants.

\subsection{Percent Body Fat (BF)}

The overall average baseline BF was $31.5 \pm 9.9 \%$. For the unconditional model there was a no significant. change over time for the group as a whole $(\mathrm{t}=-1.85, \mathrm{P}=0.07)$, though the average decline in BF was $0.24 \%$ every three months. Age was significantly related to individual differences in $\mathrm{BF}$ at baseline ( $\mathrm{t}=39.21, \mathrm{P}=0.001)$, with older participants having higher initial $\mathrm{BF}$ values. There was a significant change in $\mathrm{BF}$ over time by age $(\mathrm{t}=3.30, \mathrm{P}=0.001)$. As age increased $\mathrm{BF}$ increased over time. Gender was significantly related to 
WalkerJR et al.

individual differences in the initial BF with females being $6.47 \%$ higher than males $(t=3.38, P=0.001)$. Gender was not related to changes in $\mathrm{BF}$ over time. In the final model, age $(t=2.08, P=0.04)$ and gender $(t=6.13, P=0.0001)$ were still significant for individual differences at baseline but not related to changes in BF. Fat category was significantly related to initial values of BF. In the normal category, the initial point was $16.99 \%$ while the overweight/obese category was $29.92 \%$. Change by fat category was not signifi$\operatorname{cant}(\mathrm{t}=-1.73, \mathrm{P}=0.08)$.

\subsection{Waist Circumference (WC)}

The average initial WC for participants was $90.2 \mathrm{~cm} \pm$ 18.7. There was a significant change overall in WC from baseline to 6 months $(t=-2.41, P=0.02)$. Age was significantly related to baseline values with older participants having higher initial WC values $(\mathrm{t}=2.09, \mathrm{P}=0.04)$. Age, however, did not predict individual changes over time. Gender was also related to initial values of WC, with females lower than males $(87.5 \pm 15.3 \mathrm{~cm}$ versus $100.2 \pm 25.1$ $\mathrm{cm}$ ). Gender did not explain individual differences over time. Figure 1 shows the slope for the whole group and by gender for the WC change across the three study testing periods.

In the final model age was no longer significant. However, those in the normal BF category had significantly $(t$ $=-7.43, P=0.0001)$ smaller initial WC's than those in the overweight/obese category (20.3 cm smaller).

Figure 1. Average for WC for the Entire Group and by Gender Over the 6-Month

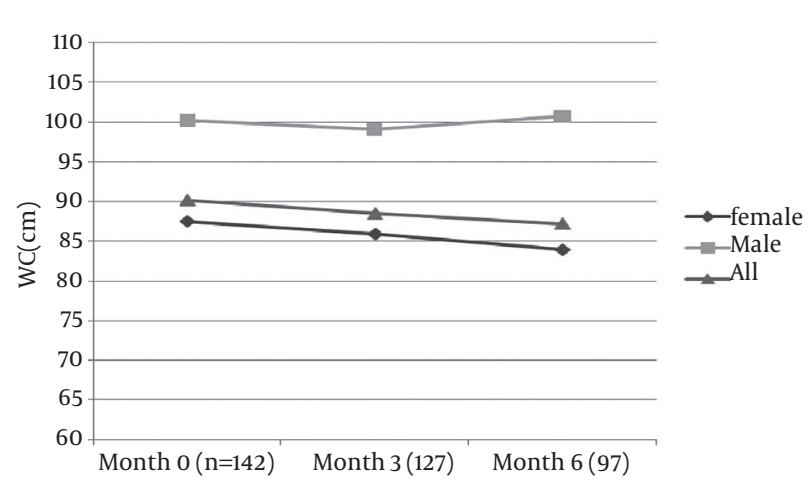

* $\mathrm{n}$ refers to the entire group.

\subsection{Sagittal Abdominal Diameter (SAD)}

On average, initial SAD for participants was $20.1 \pm 4.4 \mathrm{~cm}$. Age and gender were significantly related to individual differences in the starting values of SAD. Similar to the other body composition outcomes, older participants had higher starting SAD values than younger participants and males had a higher starting SAD value of $22.3 \pm 5.3 \mathrm{~cm}$ than females with $19.5 \pm 3.9 \mathrm{~cm}$. When all predictors were added to the model (4), there was no significant change in SAD. While there was no change in the final model, fat category was significantly related to individual differences in the starting SAD $(t=-7.79, P=0.001)$, with those in the normal fat category having a smaller SAD.

\section{Discussion}

This study evaluated the effects of a 6-month, pedometer-based walking intervention on body composition measures in the US cohort of the ASUKI Step study. We hypothesized that we would observe significant decreases in body composition measures. Contrary to our hypothesis, only minimal changes were observed in the body composition measures among the US cohort over the 6-month intervention period. Significant changes were observed for WC on the order of $-3 \mathrm{~cm}$ over the 6 month study. Female gender and higher levels of $\mathrm{BF}$ at baseline predicted greater changes in WC by end of the study. In comparison, the Swedish ASUKI Step cohort showed a small decrease in BMI $\left(-0.24 \mathrm{~kg} \cdot \mathrm{m}^{-2}\right)$ over time with a significant reduction in BMI only for older participants. Older age, a lower initial BMI value, and overfat/obese fat category in the Swedish cohort were significantly related to the linear change in BMI over time. There was a significant linear decrease in WC for Swedish participants over time with an average reduction of $0.84 \mathrm{~cm}$ every three months. Older and middle age groups and female gender predicted greater change in WC over time. Significant reductions were observed in SAD for Swedish participants over the 6 month study and the higher starting SAD values showed greater declines over time.

Even this small decrease in WC among the US cohort has clinical implications. WC is an indirect indicator of central obesity which is a strong predictor of visceral adiposity and an independent risk factor for various metabolic diseases, including type 2-diabetes (20-22). In a meta-analysis by Ohkawara and colleagues, (2008), brisk walking for 300 minutes per week had a positive impact on reducing visceral fat and improving health (23). This dose of activity is equal to 30,000 steps per week or an additional 4,285 steps per day to one's non-exercise step counts. While age, gender, or BF failed to predict changes in WC, participants in the overweight/obese categories had WC values on in the order of $20.3 \mathrm{~cm}$ higher than normal BF categories at the start of the study. This represents a sizable difference between the WC of the participants. Most cross-sectional and intervention studies using pedometers to track physical activity show an inverse relation between steps per day and WC $(8,10,24-26)$. This reinforces the value of 10,000 steps per day walking programs to reduce metabolic risk factors in adult men and women.

Interestingly, decreases in BMI observed in Swedish older adults were not observed in the US cohort. There were no significant changes in BMI over the course of the study 
and the amount of steps taken over the 6-month period did not significantly affect changes in body composition for any measure in the US cohort. It is interesting to note that the initial BMI and BF values were higher in the US cohort than the Swedish cohort. Yet, BMI and BF did not significantly change in the US cohort. Examination of the variation around the means of the body composition data indicated that the US cohort was much more heterogeneous than the Swedish cohort. This variability increases the sample required to observe statistical differences in the body composition measures; effectively lowering the power of the US cohort sample. Additionally, the sample size for the Swedish cohort was 214 and US cohort was 142, which further reduces statistical power in the US cohort. The Swedish cohort took more steps per day than the US cohort at all three measurement periods [month 1 $(13,105 \pm 2,998$ vs. $10,714.2 \pm 3,357.4)$, month $3(11,741 \pm 3,893$ vs. $8,601.8 \pm 4,954.1)$, month $6(9,939 \pm 5,949$ vs. $6,332 \pm$ 5,535.0)].

From an ecological perspective, the Swedish cohort had greater overall available resources from the natural and built environment, including access to public transportation and places that supported walking behavior. Participants in the US cohort were from a city with a population that is widely spread throughout the Phoenix valley which lacks easy and abundant access to public transportation due to the distances from home to the university worksite. Neighborhoods also do not promote an active environment as there are few walking paths and bicycle paths. In addition, the desert climate has temperatures in excess of 42 degrees Celsius in the summer months that may have contributed to the decline in steps with participants not wanting to venture outside for physical activity.

The lack of relationship between changes in BMI (reflecting body weight) and the steps taken in the US cohort may reflect a steady decline in average steps taken from the start of the study to the end (27). Average steps per day declined from 10,714 to 8,602 to 6,332 from months 1,3 , and 6 respectively. The decline may be due to either regression to the mean in step counts for the majority overweight group and/or a possible differential study drop out of those with a higher body mass. The number of participants measured at each testing session declined due to dropouts, failure to show up for measurement sessions, and/or inability to schedule participants for their measurement session (month $1, \mathrm{n}=142$; month $3, \mathrm{n}=127$; and month $6, n=97$ ) which is a retention rate of only $64 \%$ for those receiving body composition tests. Based on the physiology of weight loss, significant changes in BMI are rare when physical activity is performed at lowto-moderate intensities and with infrequent compliance to an exercise dose $(9,27)$. Since participation was not supervised and steps were self reported, it is difficult to estimate the level of adherence to the daily walking intervention. Other studies with similar community-based walking interventions show mixed results for the impact of 10,000 steps per day on BMI changes. Swartz et al. (7) show no changes in BMI in a college-age cohort of overweight women over eight weeks. De Cocker et al. (27) also reported no changes in BMI over 20 weeks in 146 participants engaged in a walking intervention. As described by Bravata et al. (9) in their meta-analysis of 26 pedometer interventions with 2,767 participants, at best decreases in BMI accompanying pedometer-based step programs are modest with a weight loss of $-0.38 \mathrm{~kg}$. No changes were observed in the SAD and BF measures in the US cohort. Possible reasons may be due to the nature of the sample or differential dropout. While the Swedish cohort noted small decreases in SAD measures, the clinical significance of these changes is hard to determine.

A major strength of the ASUKI Step study is in the innovation of the study design where teams were engaged in a social support-mediated intervention with the goal for each participant to walk 10,000 steps per day. Steps were recorded on a common website and a subsample of participants received more detailed PA monitoring, body composition, and fitness measures. The study used a quasi-experimental in design and was conducted in a community setting with online management to assess daily compliance and accuracy in recording steps. The major drawback of a quasi-experimental study with no control group is that it is not possible to isolate or describe cause and effect. In other words, interpreting the change over time should be done with caution since it cannot be determined whether changes noted were causally related to the intervention (28). We have no information regarding dietary habits such as a more energy dense diet and alcohol consumption and unhealthy lifestyle choices throughout the study and during holiday or vacation months that could affect the intervention. Other limitations of the ASUKI Step study have been reported in the previous papers $(10,16,29)$. Overall, while the dropout rate (31\% vs. $29 \%$ for US and Swedish cohort respectively) was not optimal, it was similar to other communitybased walking studies that have been reported (ranging from $25.4 \%$ - 44\%) (30-32).

We observed a $3.0 \mathrm{~cm}$ reduction in waist circumference among participants of the US cohort following the 6-month ASUKI Step 10,000 steps per day walking intervention. This decrease was independent of steps taken and observed among all participants. It is important to note that while steps did decrease throughout the study in the US cohort, the amount of steps taken at month 6 is still higher than the average steps per day taken by most Americans (5931 \pm 3664 ) (33). Compliance to the walking dose and initial physical activity and body composition levels are important to consider when studying body composition changes in such programs. As walking is a popular and easy form of exercise and transportation, engagement in health programs promoting 10,000 steps per day is an effective way to increase physical activity. While a significant change in WC, occurs, others factors may have influenced the results thus the findings are lim- 
ited.

\section{Acknowledgements}

The authors thank the data collectors, administrative support staff, and participants involved in the ASUKI Step study. ASUKI Step was funded by resources from Karolinska Institutet human resources and health promotion department, and New Lifestyles Pedometers Inc.

\section{References}

1. Committee PAGA . Physical Activity Guidelines Advisory Committee Report. In: Department of Health and Human Services, Services, DoHaH., editor. Washington (DC): 2008.

2. Haskell WL, Lee IM, Pate RR, Powell KE, Blair SN, Franklin BA, et al. Physical activity and public health: updated recommendation for adults from the American College of Sports Medicine and the American Heart Association. Med Sci Sports Exerc. 2007;39(8):1423-34

3. Ogden CL, Carroll MD, Kit BK, Flegal KM. Prevalence of obesity in the United States, 2009-2010.: US Department of Health and Human Services, Centers for Disease Control and Prevention, National Center for Health Statistics; 2012.

4. Lee CD, Blair SN, Jackson AS. Cardiorespiratory fitness, body composition, and all-cause and cardiovascular disease mortality in men. Am J Clin Nutr. 1999;69(3):373-80.

5. Services TG to CP. . The Community Guide - Worksite Health Promotion. 14 Jun, 2013. Available from: http://www.thecommunityguide.org/worksite/index.html.

6. Van Dyck D, De Greef K, Deforche B, Ruige J, Tudor-Locke CE, Kaufman JM, et al. Mediators of physical activity change in a behavioral modification program for type 2 diabetes patients. Int $J$ Behav Nutr Phys Act. 2011;8:105.

7. Swartz AM, Strath SJ, Bassett DR, Moore JB, Redwine BA, Groer $M$, et al. Increasing daily walking improves glucose tolerance in overweight women. Prev Med. 2003;37(4):356-62.

8. Freak-Poli RL, Wolfe R, Walls H, Backholer K, Peeters A. Participant characteristics associated with greater reductions in waist circumference during a four-month, pedometer-based, workplace health program. BMC Public Health. 2011;11:824.

9. Bravata DM, Smith-Spangler C, Sundaram V, Gienger AL, Lin N, Lewis R, et al. Using pedometers to increase physical activity and improve health: a systematic review. JAMA. 2007;298(19):2296-304.

10. Soroush A, Walker J, Poortvliet E, Belyea M, Ainsworth BE, Swan $\mathrm{PD}$, et al. The effects of a 6-month pedometer-determined physical activity intervention on body composition characteristics in Swedish adults: The ASUKI Step study. Int J Body Compos Res . 2012;10(2).

11. Marshall SJ, Levy SS, Tudor-Locke CE, Kolkhorst FW, Wooten KM, Ji M, et al. Translating physical activity recommendations into a pedometer-based step goal: 3000 steps in 30 minutes. Am J Prev Med. 2009;36(5):410-5.

12. Gilson N, McKenna J, Cooke C, Brown W. Walking towards health in a university community: a feasibility study. Prev Med. 2007;44(2):167-9.

13. Harvey JT, Eime RM, Payne WR. Effectiveness of the 2006 Commonwealth Games 10,000 Steps Walking Challenge. Med Sci Sports Exerc. 2009;41(8):1673-80.

14. Murphy MH, Murtagh EM, Boreham CA, Hare LG, Nevill AM. The effect of a worksite based walking programme on cardiovascular risk in previously sedentary civil servants [NCT0 0284479]. BMC Public Health. 2006;6:136.
15. Murphy MH, Nevill AM, Murtagh EM,Holder RL. The effect of walking on fitness, fatness and resting blood pressure: a meta-analysis of randomised, controlled trials. Prev Med.2007;44(5):377-85.

16. Ainsworth BE, Der Ananian C, Soroush A, Walker J, Swan P, Poortvliet E, et al. "ASUKI Step" pedometer intervention in university staff: rationale and design. BMC Public Health. 2012;12:657.

17. Ritchie JD, Miller CK, Smiciklas-Wright H. Tanita foot-to-foot bioelectrical impedance analysis system validated in older adults. J Am Diet Assoc. 2005;105(10):1617-9.

18. Gallagher D, Heymsfield SB, Heo M, Jebb SA, Murgatroyd PR, Sakamoto Y. Healthy percentage body fat ranges: an approach for developing guidelines based on body mass index. Am J Clin Nutr. 2000;72(3):694-701.

19. Cohen J, Cohen P. Applied Multiple Regression/correlation Analysis for the Behavioral Sciences.: L. Erlbaum Associates; 1983.

20. Janssen I, Katzmarzyk PT, Ross R. Waist circumference and not body mass index explains obesity-related health risk. Am J Clin Nutr. 2004;79(3):379-84.

21. Janssen I, Heymsfield SB, Allison DB, Kotler DP, Ross R. Body mass index and waist circumference independently contribute to the prediction of nonabdominal, abdominal subcutaneous, and visceral fat. Am J Clin Nutr. 2002;75(4):683-8.

22. Sowers JR. Obesity as a cardiovascular risk factor. Am J Med. 2003;115 Suppl 8A:37S-41S.

23. Ohkawara K, Tanaka S, Miyachi M, Ishikawa-Takata K, Tabata I. A dose-response relation between aerobic exercise and visceral fat reduction: systematic review of clinical trials. Int J Obes (Lond). 2007;31(12):1786-97.

24. Thompson DL, Rakow J, Perdue SM. Relationship between accumulated walking and body composition in middle-aged women. Med Sci Sports Exerc. 2004;36(5):911-4.

25. Maturi MS, Afshary P, Abedi P. Effect of physical activity intervention based on a pedometer on physical activity level and anthropometric measures after childbirth: a randomized controlled trial. BMC Pregnancy Childbirth. 2011;11:103.

26. Musto A, Jacobs K, Nash M, DelRossi G, Perry A. The effects of an incremental approach to 10,000 steps/day on metabolic syndrome components in sedentary overweight women. J Phys Act Health. 2010;7(6):737-45.

27. De Cocker KA, De Bourdeaudhuij IM, Cardon GM. The effect of a multi-strategy workplace physical activity intervention promoting pedometer use and step count increase. Health Educ Res. 2010;25(4):608-19.

28. Middel B, van Sonderen E. Statistical significant change versus relevant or important change in (quasi) experimental design: some conceptual and methodological problems in estimating magnitude of intervention-related change in health services research. Int J Integr Care. 2002;2.

29. Soroush A, Der Ananian C, Ainsworth BE, Belyea M, Poortvliet E, Swan PD, et al. Effects of a 6-Month Walking Study on Blood Pressure and Cardiorespiratory Fitness in U.S. and Swedish Adults: ASUKI Step Study. Asian J Sports Med. 2013;4(2):114-24.

30. Bassett DJ, Wyatt HR, Thompson H, Peters JC, Hill JO. Pedometermeasured physical activity and health behaviors in U.S. adults. Med Sci Sports Exerc. 2010;42(10):1819-25.

31. Chan CB, Ryan DA, Tudor-Locke C. Health benefits of a pedometer-based physical activity intervention in sedentary workers. Prev Med. 2004;39(6):1215-22.

32. Faghri PD, Omokaro C, Parker C, Nichols E, Gustavesen S, Blozie E. E-technology and pedometer walking program to increase physical activity at work. J Prim Prev. 2008;29(1):73-91.

33. Tudor-Locke C, Ham SA, Macera CA, Ainsworth BE, Kirtland KA, Reis JP, et al. Descriptive epidemiology of pedometer-determined physical activity. Med Sci Sports Exerc. 2004;36(9):1567-73. 